\section{Recent applications of}

\section{Synchrotrons in cancer}

\section{therapy with Carbon}

\section{lons}

Ugo Amaldi ${ }^{1}$, Gerhard Kraft ${ }^{2}$,

${ }^{1}$ University of Milano Bicocca and TERA Foundation

${ }^{2}$ GSI and Technical University, Darmstadt

A s shown in Table 1, accelerators originally designed for scientific research have found a large number of industrial and medical applications.

The main medical applications besides radioactive isotope production with cyclotrons are the electron linacs of a few $\mathrm{MeV}$ for radiotherapy of deep seated tumours. Every year in the developed countries about 40,000 people out of 1 million inhabitants are diagnosed as having cancer. About $50 \%$ of these patients are treated with radiation, mainly high energy photons produced by electron linacs. In $90 \%$ of the cases the electron beam is converted to Bremsstrahlung photons. These photon beams (called "X-rays" by medical doctors) have replaced the low energy X-rays and the ${ }^{60} \mathrm{Co}$ gamma radiation because of a better depth-dose distribution (Fig. 1). Due to the fact that Compton-electrons go forward with a few centimetre range, the dose increases at the entrance (build-up effect) to a maximum value at $3-4 \mathrm{~cm}$ below the skin. This increase is followed by a shallow exponential decay for greater depth.

\begin{tabular}{|l|r|}
\hline \multicolumn{1}{|c|}{ Application } & \multicolumn{1}{|c|}{$\begin{array}{c}\text { Number } \\
\text { in use }\end{array}$} \\
\hline High-energy accelerators for research $(\mathrm{E}>1 \mathrm{GeV})$ & 120 \\
\hline $\begin{array}{l}\text { Low energy accelerators for research } \\
\text { (including biomedicine) }\end{array}$ & $\sim 1000$ \\
\hline Synchrotron radiation sources & $>100$ \\
\hline Medical radioisotope production & $\sim 200$ \\
\hline Radiotherapy accelerators & $>7500$ \\
\hline Accelerators for industrial processing and research & $\sim 1500$ \\
\hline $\begin{array}{l}\text { Ion implanters, modification of surfaces and } \\
\text { matter in bulk }\end{array}$ & $>7000$ \\
\hline
\end{tabular}

$\triangle$ Table 1: World accelerators running in 2003 [1].

In the most advanced treatments the X-ray dose is given from many (5-10) ports by rotating the electron linac around the patient and modulating the shape and intensity of each port separately using computer-controlled multileaf-collimators. With this Intensity Modulated Radio Therapy (IMRT) the most conformal $\mathrm{X}$-ray treatment can be given at the expense of a greater integral dose, which is nevertheless distributed to the normal tissues around the target.

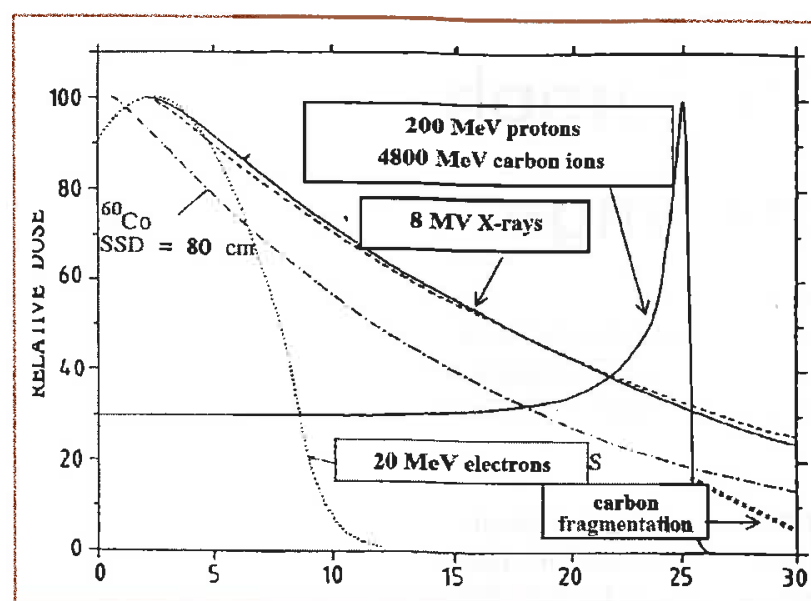

A Fig. 1: Depth dependence of the deposited dose for different radiations.

As the induction of secondary tumours is rather a volume than a dose effect, a better cure with lower risk becomes possible when the physical properties of the radiation are changed. This is possible by the transition from X-rays to ion beams such as protons or carbon.

The depth-dose curves of proton and light ion beams are completely different from those of $\mathrm{X}$-rays because these charged particles have little scattering in matter and give the highest dose near the end of their range in the 'Bragg peak' (Fig. 1). Often they are said to have an 'inverse' distribution of the longitudinal energy deposition with respect to $\mathrm{X}$-rays. Protons and carbon ions were first proposed for radiotherapy applications by R.R. (Bob) Wilson in 1946 [2].

The energies for reaching deep-seated tumours (about $25 \mathrm{~cm}$ of water equivalent) are $200 \mathrm{MeV}$ for protons and $4800 \mathrm{MeV}$ for carbon ions, 24 times larger. Protons beams are obtained either from cyclotrons (normal or superconducting) or from synchrotrons having a diameter of 6-7 metres. Only synchrotrons are used to produce carbon ions of about $400 \mathrm{MeV}$ per nucleon $(400 \mathrm{MeV} / \mathrm{u})$. Their magnetic rigidity of approximately $6 \mathrm{Tm}$ is about three times larger than that of $200 \mathrm{MeV}$ protons so that about 20 metre diameter synchrotrons are needed when fields in the range 1.5-2 Tesla are used. Superconducting cyclotrons are an option that is at present considered also for ion therapy.

For mono-energetic ions the Bragg peak, shown in Fig. 1, is very narrow, so that the energy of the particles has to be changed during the irradiation to cover the tumour depth. In cyclotrons the beam energy cannot be varied, so that movable energy absorbers and magnetic selection systems have to be used to adapt the range of the particles to the depth of the target to be irradiated. In synchrotrons it is easy to vary the energy of the extracted beam.

Until 1997 relatively simple 'passive spreading systems' have been used to produce a Spread Out Bragg Peak (SOBP) in all hadrontherapy centres. In this approach, a first 'scatterer' widens the pencil beam while their energy is adapted to the distal form of the tumour by using appropriate absorbers. Downstream of the single (and sometimes double) scatterer, the transverse form of the irradiation field is defined by collimators. Only in 1997 at GSI [3] and PSI [4] have the novel 'active spreading systems' been developed where the charged hadrons are magnetically guided over the treatment area and modulated in intensity (Intensity Modulated Particle Therapy = IMPT). 
In this 'active spreading' technique the target volume is divided into slices of equal particle energy and each slice is divided into small volumes called 'spot' or 'voxels' (i.e. 3D pixels) that are treated separately by moving transversally the beam by means of bending magnets. When one slice has been treated, the energy of the beam is reduced for the next slice. In practice, the complete target volume consists of $10,000-30,000$ voxels which are treated in 2-6 minutes. All recent hadrontherapy facilities have the possibility of using active systems. Most existing facilities still use protons delivered in fixed horizontal beam lines, combined with passive scattering systems.

From the end of the century the newly built protontherapy centres feature isocentric 'gantries' to improve the conformity of the treatment, avoiding high doses to healthy tissues by rotating the therapeutic beam around the patient as it is done in all X-ray treatments. The magnetic rigidity of $200 \mathrm{MeV}$ protons is such that a standard magnetic channel capable of doing so has a typical radius of $4-5 \mathrm{~m}$.

In a conventional treatment with X-rays a total dose of 60-70 Gy $(1 \mathrm{~Gy}=1 \mathrm{~J} / \mathrm{kg})$ is deposited in a tumour target in typically 30 fractions over six weeks to give time for re-oxygenation of hypoxic - and therefore radioresistant - tumour cells and for the transition of tumour cells from radio-resistant cell cycle stages to more sensitive stages. In addition the unavoidably irradiated healthy cells have a chance to repair the radiation damage. Typically a proton treatment requires 20 fractions and allows higher doses to the tumour. It has to be remarked that a larger dose is beneficial because even a modest $10 \%$ increase of the dose deposited in a tumour gives typically an increased probability of local control of the tumour itself by about $20 \%$. This implies that passing from $60 \mathrm{~Gy}$ to $66 \mathrm{~Gy}$, for instance, the control probability increases from $50 \%$ to $60 \%$, a not negligible gain. This fact is independent of any clinical trial and is the strongest argument in favour of protontherapy: since proton and X-rays beams produce practically the same biological effects on the irradiated cells, a better spatial distribution immediately translates either in a reduction of the side effects or in an increase of the tumour control probability.

Since good review articles on protontherapy have been recently written by E. Pedroni for Europhysics News [5] and by M. Goitein et al for Physics Today [6], here we limit ourselves to remark that - by the beginning of 2005 - about 40,000 patients had been treated with proton beams and that five commercial companies offer turnkey centres of proton therapy, two based on cyclotrons and three on synchrotrons, all of them featuring isocentric 'gantries': ACCEL, IBA, Hitachi, Mitshubishi and Optivus.
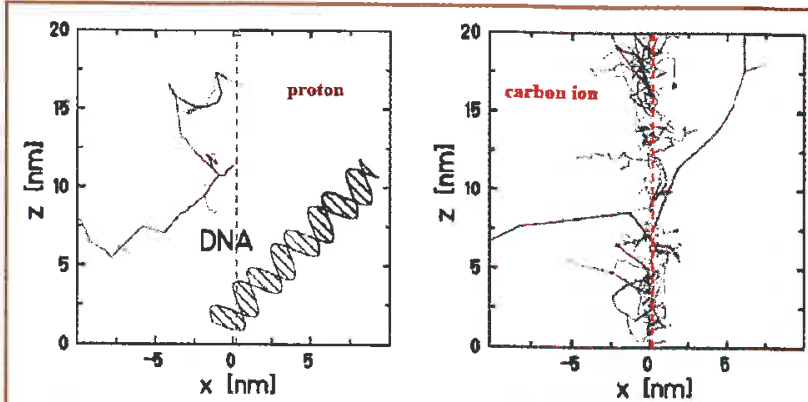

4Fig. 2: The structure of a proton and a carbon track in nanometre resolution are compared to a schematic representation of a DNA molecule. The density of ionizations and delta-rays are different because in each cell a carbon ion leaves 24 times more energy than a proton of the same range.

By the end of 2004 there were about fifteen hospital-based protontherapy centres running or under construction in the world [7]. This single number justifies the statement that protontherapy is booming.

\section{The rationale for Carbon Ion Therapy}

Because of smaller scattering in both the lateral and the longitudinal direction, carbon beams exhibits dose gradients three times steeper than protons. But the main reason for the transition to carbon ions is the increased Relative Biological Effectiveness (RBE) in the last few centimetres of the carbon range. (RBE is defined as the ratio of the photon dose to the particle dose necessary to produce the same biological effect, e.g. inactivation of $90 \%$ of the irradiated cells.)

Dose is a macroscopic parameter that does not describes the microscopic structure of the energy deposition events. It is the spatial distribution of the ionizations around the particle trajectory - called the 'track structure' - that determines the biological effect. The relevant scale is the diameter of the DNA molecule, the main target of the radiation attack inside the cell nucleus (Fig. 2).

Because of its importance the DNA molecule is protected by an elaborate repair system that restores with high fidelity the base damages, the single- and most of the double strand breaks. In the tracks of carbon ions the local ionisation density and hence the density of severe DNA lesions becomes so high that repair fails (clustered multiply-damaged sites). Then the reproduction of the tumour cells is hindered and the tumour stops to grow. In many cases the cell internal program for its own destruction (apoptosis) is activated yielding a fast tumour regression.
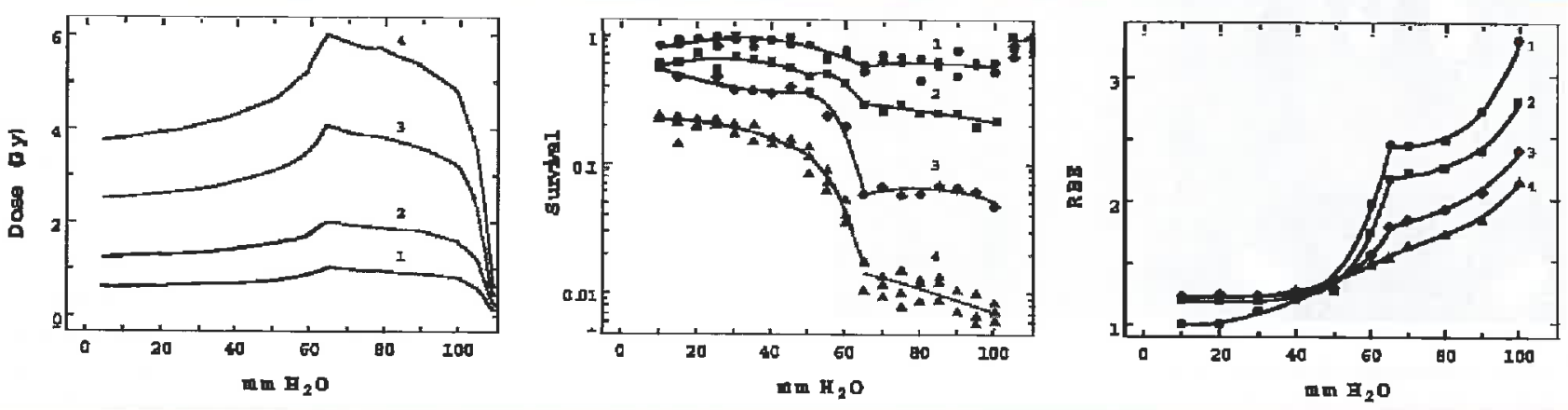

Aig. 3: Comparison of the physical absorbed dose (left panel) and measured cell survival of CHO cells (central panel) in a Spread Out Bragg Peak for various dose distributions. RBE values calculated from the measured cell survival are shown in the right panel. [8]. 
In the 'entrance channel' of a carbon beam, due to the plateau of the energy deposition, the density of severe lesions is lower and DNA damage can be mostly repaired as is in the case of X-rays and protons. Thus for carbon ions the increase in RBE is significant mainly over the Spread Out Bragg Peak (SOBP) (Fig. 3). Since the RBE varies along the SOBP the physical dose has to decrease towards the distal edge in order to achieve a homogeneous biological effect over the simulated tumour volume.

By contrast, for protons only over the last few micrometers is the RBE definitely larger than 1 . For this one can state that protons have the same radiobiological effect as X-rays.

Because of the high effectiveness in suppressing the repair, heavy ion beams are most suited for slowly growing, well repairing tumours, which are precisely those tumours that are resistant to photons and protons. In these cases, the biological effect at the end of the range is increased 3-4 times compared to conventional radiations. In summary, carbon ion beams of about $400 \mathrm{MeV} / \mathrm{u}$ are indicated for treatment of deep-seated tumours, which are radio-resistant both to X-rays and to protons. These types of tumours are thus the elective targets in a carbon ion facility. Proton therapy is well adapted to the cases where a greater tumour dose is needed but where the tumour is not too close to organs at risk because protons have a dose gradient, always 3 times greater than carbon. These arguments are important since a proton therapy facility is about $30 \%$ cheaper than a combined proton / carbon ion centre, which requires a total investment cost of about $100 \mathrm{ME}$.

$\mathrm{RBE}$ is a complex function of the biological response to the microscopic structure of the radiation field, i. e. to the radiation quality. Consequently, $\mathrm{RBE}$ changes over the treatment area since the radiation quality changes (as indicated by the fragmentation tail of figure 1) and RBE is different for different tissues of different repair capacity. For a quantitative calculation of the RBE effects, a theoretical model, the Local Effect Model (LEM) has been developed at GSI [9].

In the LEM approach the individual RBE of the tumour to be treated (and the healthy tissues to be avoided) is not derived from in vitro data, but is determined from the intrinsic repair capacity as given by the dose response curve of the same tissue to photons. The very successful experience of the more than two hundred patients treated up to now at GSI using this treatment planning fully confirms the basic rationale of these calculations. The IMPT plan of Fig. 4 has been obtained by making use of a code based on LEM. In this case, and in most others, the advantage of an inverse dose profile, with the high dose and high LET at the end of the range, allows reducing the dose to the normal tissues outside the target volume by a factor of 2-3.

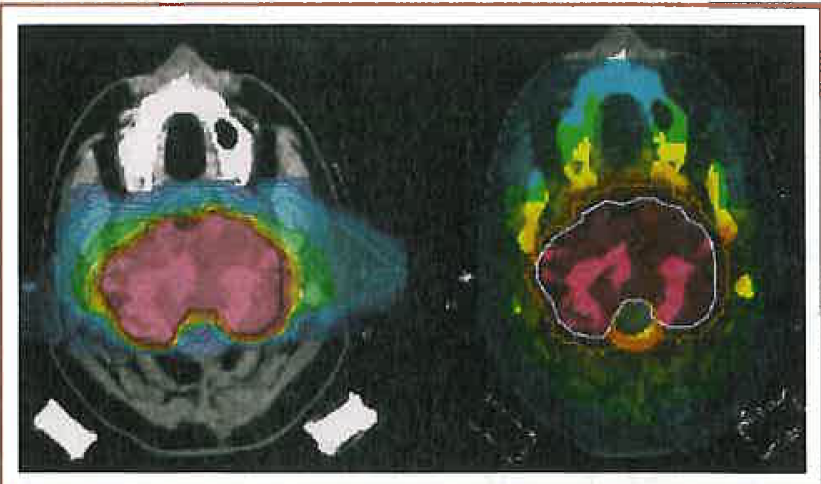

A Fig. 4: Comparison of treatment plans with 2 fields of carbon ion (IMPT - left panel) and with 9 fields of X-rays (IMRT - right panel). In both cases the conformity to the target volume is good but for carbon ions the dose to the normal tissues is much smaller.
A further advantage of particle beams, and especially of ions like carbon, is the 'in situ' production of positron emitters such as ${ }^{10} \mathrm{C}$ and ${ }^{11} \mathrm{C}$ and ${ }^{15} \mathrm{O}$. Because the stripping of one or two neutrons is a minor perturbation, the residual carbon ions form a maximum of $\beta^{+}$activity close to the Bragg maximum of the stable carbon ions. By monitoring the positron emitting isotopes by a PET camera during and shortly after the beam application (Fig. 5), the actual stopping points of the beam can be controlled. PET control gives - for the first time in 110 years of radiotherapy - an 'in situ' control of the treated field that also checks all the calculations and calibrations of the energy losses used for treatment planning. The online PET technique has been developed by FZR (Dresden) [10].

\section{Clinical Results and numbers of potential patients}

In 1994 the first patient was treated with carbon ions at NIRS in Japan where the construction of HIMAC (Heavy Ion Medical Accelerator in Chiba - www.nirs.go.jp) was promoted by Y. Hirao. At HIMAC the choice was made not to construct rotating gantries but to have a horizontal and a vertical beam in a single treatment room. The other two rooms feature horizontal beams. By 2005 more than 2,000 patients had been treated under the leadership of $\mathrm{H}$. Tsujii and very promising results have been obtained [11].

Patients with early-stage Non-Small-Cell Lung Cancers (NSCLC) that could not be operated on have been treated with different dose-fractionation schedules: 18 fractions in 6 weeks, 9 fractions in 3 weeks and 4 fractions in 1 week. For the shortest one the local control rate was $73 \%$ at 3 years. At present a Phase I/II study is in progress by treating these patients with a single dose of 28 Gye delivered through four ports. Short schedules have also being studied for hepatocellular carcinomas: in the last phase II study the 3-year local control rate was $90 \%$ using a 4-fraction/1 week regimen. Finally, with $70 \mathrm{GyE}$ in 16 fractions the local tumour control rate for bone and soft tissue sarcomas - which are very radioresistant tumours - was $88 \%$ after 3 years and the 3 -year survival rate was $54 \%$.

Almost 250 patients have been irradiated at GSI with a horizontal carbon beam and the active spreading system described above. Starting in 1988 one of us (G.K.), together with G. Gademann and G. Hartmann, proposed a two-step project: installation of an irradiation unit for experimental patient treatment at the new heavy ion accelerator SIS of GSI and, as a second phase, the construction of a dedicated heavy ion therapy unit at the Heidelberg clinic. In the summer of 1993 the construction of the therapy cave started and in December 1997 the first two patients were treated. In 2003 the construction of the Heidelberg Ion Therapy HIT was initiated.

At Darmstadt 3 year tumour-control rates for chordomas and chondrosarcomas, which were a subsection of the large variety of tumours treated at NIRS, are $100 \%$ and $84 \%$ respectively [12]. These values are significantly better than reported for conventional radiotherapyand are also based on an accelerated fractionation scheme of 20 fractions in 20 days.

As far as the number of potential patients is concerned, detailed analysis have been made in Germany, Italy, Austria and France by groups of radiotherapists who have applied to the national data specific criteria for each tumour site. The results of these different approaches are very consistent. As an overall summary it can be stated that about $1 \%$ of the patients today treated with $\mathrm{X}$ rays must be irradiated with protons since the outcomes are definitely better than those of conventional therapy; about $12 \%$ of the X-ray patients would profit from a proton treatment but further clinical 


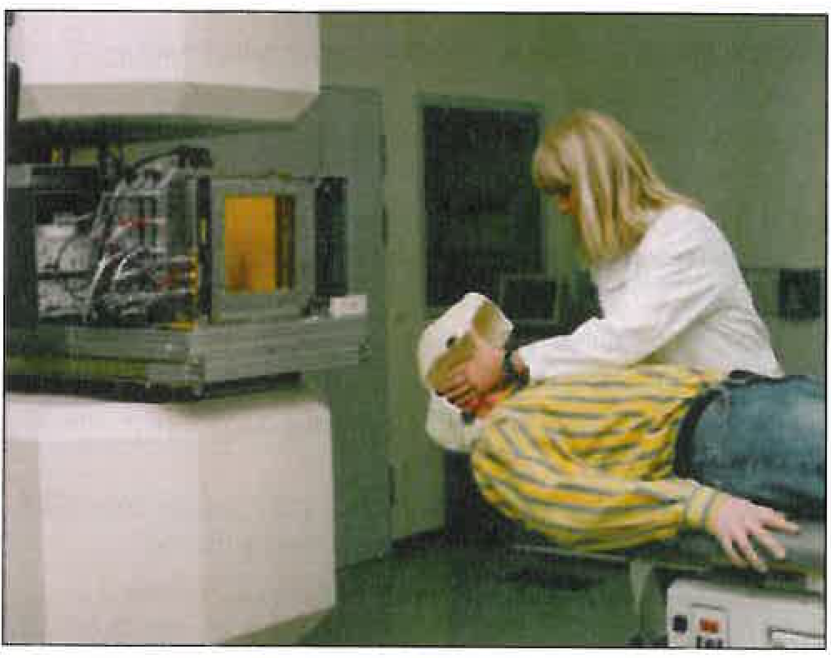

$\triangle$ Fig. 5: The GSI horizontal beam has been used to treat mainly intracranial and head and neck tumours. The two white boxes, placed above and below the patient, contain the detectors for the on-line PET measurement of the stopping particles.

trials are needed to quantify, site by site, the clinical advantages; about $3 \%$ of the $\mathrm{X}$ ray patients would profit from carbon-ion therapy, but many more dose-escalation studies and clinical trials are needed.

Overall, $15 \%$ of the about 20,000 patients out of every 10 million inhabitants treated with conventional radiation would receive a better treatment with hadron beams. If the actual average recruitment rate could be as large as $50 \%$, these figures would require a proton therapy centre (treating 1500 patients a year) for every 10 million people and a carbon ion centre for every 50 million people. This is indeed the conclusion reached by the Italian association for radiotherapy and oncology AIRO [13].

As far as costs are concerned, it has been said that proton treatment costs 2-3 times more than conventional treatment [14]. The economy of carbon treatment is different. The possible shortening of the treatment to less than 10 fractions is a great advantage for a very effective use of the costly infrastructures and - if confirmed by the ongoing clinical trials - will reduce the cost of the treatments and may become one of the main reasons behind the rapid diffusion of light ion therapy in the future. At present the German health insurance is negotiating 15,000 to $20,000 €$ per patient for particle treatment.

\section{European Carbon Ion facilities and enlight}

Based on the successes of the pilot project, the Heidelberg Ion Therapy Centre HIT (Fig. 6) was approved in 2001 and the civil engineering work could start in November 2003 [15]. The total estimated cost of $90 \mathrm{M} €$ is shared equally between the federal Government and a bank loan of the Heidelberg hospital. The first treatment should take place in 2007.

HIT is an ambitious project that applies all the techniques and methods developed in the framework of the Darmstadt pilot project and features the first carbon ion gantry, which weighs about 600 tons.

At the end of 1995 one of us (U.A) - with the help of M. Regler - drew the interest of the CERN management to the design of an optimized synchrotron for light ion (and proton) therapy. PIMMS (Proton Ion Medical Machine study) was a collaboration of CERN, Med-AUSTRON (Austria), and TERA (Italy) [16].
The second European centre is being built in Pave: CNAO (the "Centro Nazionale di Adroterapia Oncologica") and has been designed by the TERA Foundation simplifying the PIMMS project. In the PIMMS/TERA design (Fig. 7) the sources are inside the ring together with a single $7 \mathrm{MeV} / \mathrm{u}$ injector of the GSI design [17].

In 2001 the Italian Government created the CNAO Foundation with five large hospitals of the Lombardy region and TERA as founders. In September 2003 TERA completed the technical drawings and the specifications, which have been worked out in collaboration with INFN, CERN and GSI. Since 2003 INFN is a participant of the foundation and has taken major responsibilities in the construction of the Centre. The facility is foreseen to be ready by the end of 2007.

In Europe the construction of other carbon ion centres are under consideration: Med-Austron in Wiener Neustadt, ETOILE in Lyon, ASCLEPIOS in Caen and a centre proposed by the Karolinska Institute for Sweden. These projects have adopted for their proposal the PIMMS/TERA design but the technical choices will be frozen when the financial framework is defined.

In 1998 the Med-Austron team presented to the Austrian authorities their project. At the end of 2004 the Austrian Government, the State of Lower-Austria and the town of Wiener Neustadt granted a substantial part of the required funding and initiated the creation of the organizational structure to advance the project. In 1998 the University Claude Bernard of Lyon commissioned a preliminary examination of a hadrontherapy centre based on the TERA design. A proposal centered on the health care and the economic aspects of the Lyon project was then issued. In 2004 ASCLEPIOS, a similar project at Caen, was proposed. In May 2005 the French Health Minister announced that the first French Ion Therapy centre will be built in Lyon.

The European projects (sited in Heidelberg, Pave, Wiener Neustadt, Lyon, Caen and Stockholm) have teamed with ESTRO (the European Society for Radiotherapy), EORTC (the European Organization for Cancer Research), CERN and GSI to form the European Network for Light Ion Therapy. In 2002 ENLIGHT was financed for three years by the European Union [18]. The work

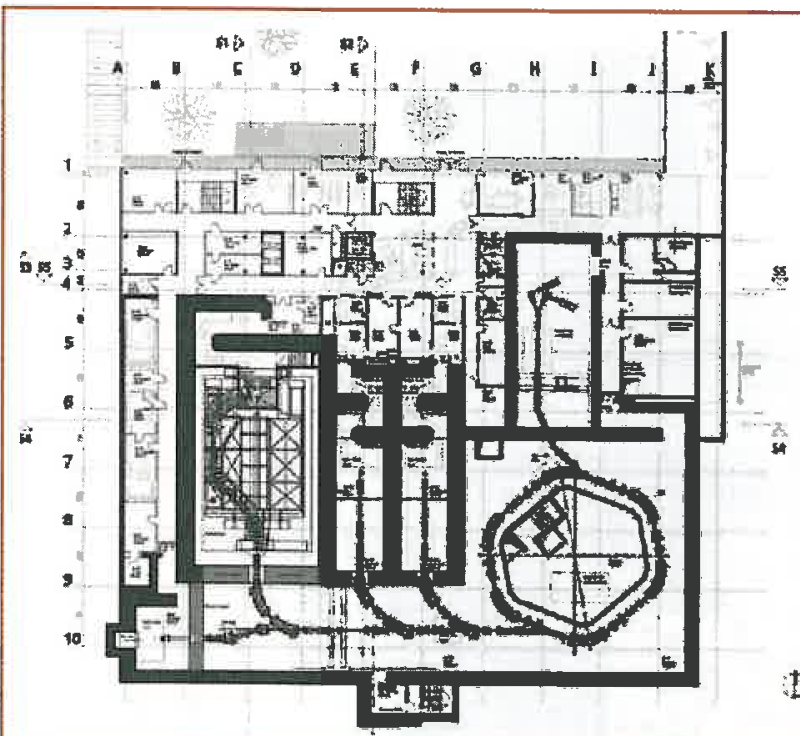

Fig. 6: The Heidelberg facility HIT (Heidelberg lon Therapy) features three treatment rooms. One of them hosts a rotating carbon ion gantry of new design. A single $7 \mathrm{MeV} / \mathrm{u}$ linac injects in the synchrotron both protons and carbon ions. 
done and the existence of this network, and of its potential successor, guarantees that the future of carbon ion therapy in Europe is on a good track and that the foreseen facilities will be run for the benefit of all European patients.

Finally it should be mentioned that the industry has meanwhile shown its interest in the forthcoming market of heavy-particle therapy. As stated above, five companies are selling proton therapy units. In the heavy-ion market Mitsubishi has designed a "micro HIMAC", a synchrotron for combined proton and carbon therapy and Siemens Medical Solutions has designed a combined proton - carbon facility on the basis of exclusive licenses of the GSI patents and know-how. IBA and ACCEL are studying superconducting cyclotrons for carbon ions and other companies are organizing themselves to enter this promising market. The strong interest of industrial companies in ion therapy indicates the large potential of this novel strategy for combating cancer.

\section{About the authors:}

Ugo Amaldi, CERN senior scientist and spokesman of the DELPHI collaboration at LEP created in 1992 the TERA Foundation and is now professor of Medical Physics at the University of Milano Bicocca.

Gerhard Kraft worked at the Berkeley ion therapy facility and started the biophysics activities at GSI in 1976. He is now head of the department of biophysics at GSI and professor at TU Darmstadt and GH Kassel.

The authors share their interest in promoting ion therapy because of the expected benefit for many European patients.

\section{Acknowledgements}

One of us (U.A.) is very grateful to the Monzino Foundation (Milan) for the continuous and generous support given to TERA activities.

\section{References}

[1] W. Maciszewski, W. Scharf, Eksploatacja i NiezawodnoE€̧, No. 4, (2003), 4.

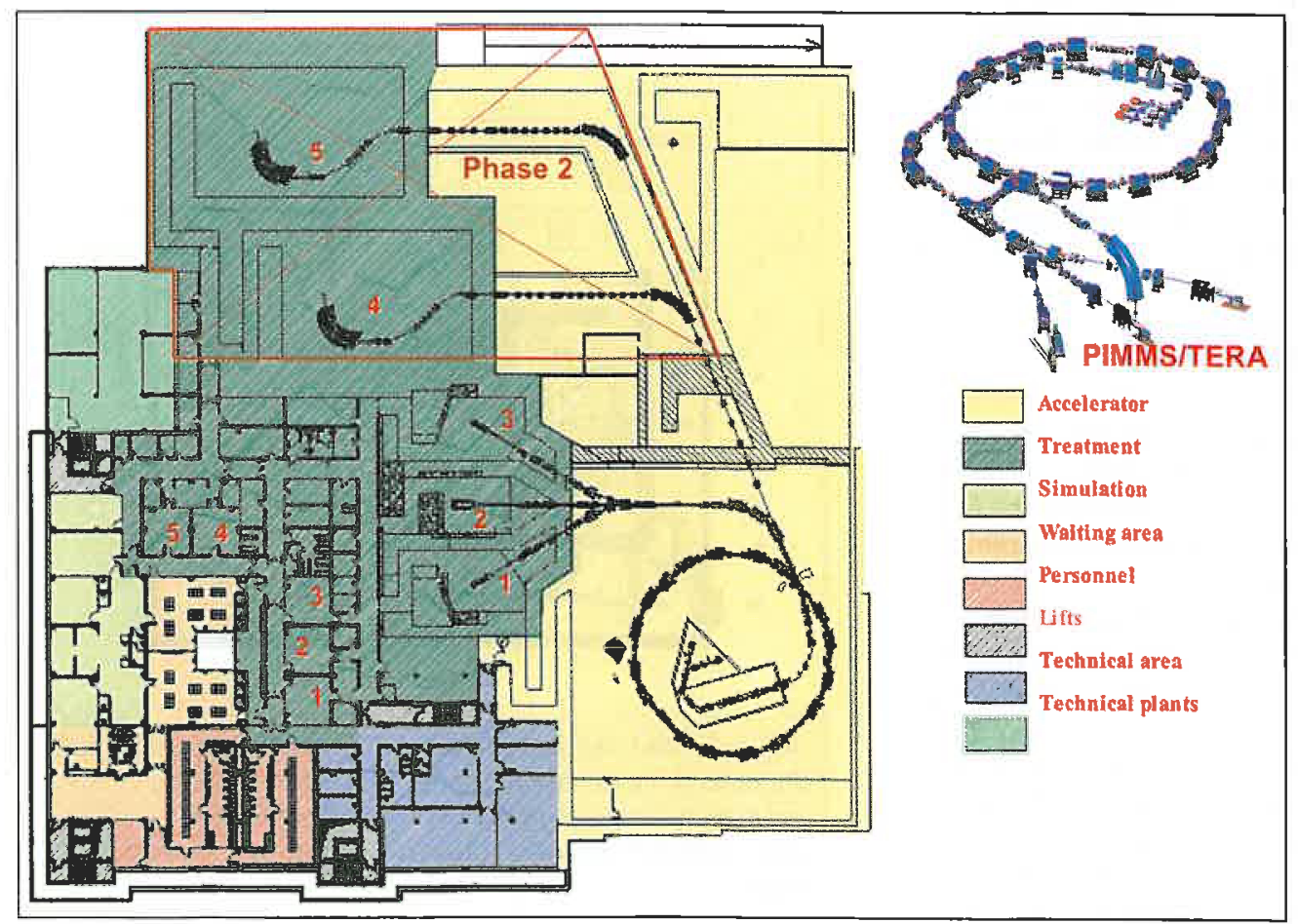

[2] R.R. Wilson, Radiological use of fast protons, Radiobiology 47 (1946) 487-491.

[3] T. Haberer et al, Magnetic scanning system for heavy ion therapy, Nudl. Instrum. Methods Phys. Res. A330 (1993) 296-314.

[4] E. Pedroni et al, The $200 \mathrm{MeV}$ proton therapy project at the Paul Scherrer Institute: conceptual design and practical realisation, Med. Phys .22 (1995) 37-53.

[5] E. Pedroni, Will we need proton therapy in the future?, Europhysics News 31/6 (2000) 18-22.

[6] M. Goitein, A. Lomax, E. Pedroni, Treating cancer with protons, Phys. Today, 55/9 (2002) 45-50.

[7] Particles Newsletter, No. 35 , Sisterson J. (Ed.), January 2005. Information on all the centres can be found visiting the site:

[8] G. Kraft et al, What kind of radiobiology should be done at a Hadrontherapy centre?, in Advances in Hadrontherapy, U. Amaldi, B. Larsson and Y. Lemoigne Eds., Elsevier, Amsterdam-LausanneNew York-Oxford-Shannon-Tokyo, 1997, pp. 38-54.

[9] M. Scholz, G. Kraft, Calculation of Heavy Ion Inactivation Probabilities Based on Track Structure, X-ray sensitivity and target size, Radiat. Prot. Dosimetry 52 (1994) 29-34.

[10] W. Enghardt, The spatial distribution of positron-emitting nuclei generated by relativistic light ion beams in organic matter, Phys. Med. Biol. 37 (1992) 2127-2131.

[11] H. Tsujii et al, Overview of clinical experience on carbon ion therapy at NIRS, Radiotherapy and Oncology 73/2 (2004) S41-S49.

[12] D. Schulz-Ertner et al, Results of carbon ion radiotherapy in 152 patients, Int. J. Radiation Biol. Phys., 58 (2004) 631 - 640.

[13] M. Krengli, R. Orecchia, Medical aspects of the National Centre for Oncological Hadrontherapy (CNAO) in Italy, Radiotherapy and Oncology 73/2 (2004) S21-23.

[14] M. Goitein, M. Jermann, The relative costs of proton and X-ray radiation therapy, Clin. Oncol. 15/1 (2003) \$37-50.

[15] Proposal for a Dedicated Ion Beam Facility for Cancer Therapy, J. Debus, K.D. Gross and M. Pavlovic Eds, GSI, Darmstadt, 1998.

[16] L. Badano, M. Benedikt, P.J. Bryant, M. Crescenti, P. Holy, P. Knaus, A. Meier, M. Pullia, S. Rossi, Proton-Ion Medical Machine Study (PIMMS). Part I and II, CERN/PS 1999-010 DI and CERN/PS 2000-007 DR, Geneva.

[17] The Path to the Italian National Centre for Ion Therapy U. Amaldi Ed., Mercurio, Vercelli, 2005. In print.

[18] For the Meeting closing the three year ENLIGHT activity Oropa, Italy, June 2005 - see the sites www.tera.it and www.iseonline.tera.it.

Fa. 7 : Layout of the CNAO underground floor. At the beginning the bunker will not feature the two very large gantry rooms that will be built and equipped with gantries at a later stage. 